\title{
Molecular design of metalloproteins
}

\section{P201}

\section{Non-heme iron complexes as efficient bleomycin mimics} Qian Li, Gerard Roelfes

Stratingh Institute for Chemistry, University of Groningen,

9747 AG Groningen, The Netherlands. Q.Li@rug.nl

Bleomycin (BLM) is a glycopeptide anticancer drug that effectively carries out single and double strand DNA cleavage [1]. In our group, $\mathrm{N}, \mathrm{N}$-bis(2-pyridylmethyl)- $\mathrm{N}$-bis(2-pyridyl)methyl amine (N4Py) was designed as a mimic for the metal binding domain of BLM [2]. Its corresponding iron(II) complex was capable of effective single strand DNA cleavage. A significant increase in cleavage activity was observed by the introduction of a DNA binding moiety, i.e., 9-aminoacridine [3, 4]. Here, we will present studies aimed at further increasing the DNA cleavage activity by variation of the DNA binding moiety and the spacer. Furthermore, it will be shown that the activity of these iron complexes could be increased further by irradiation with light.
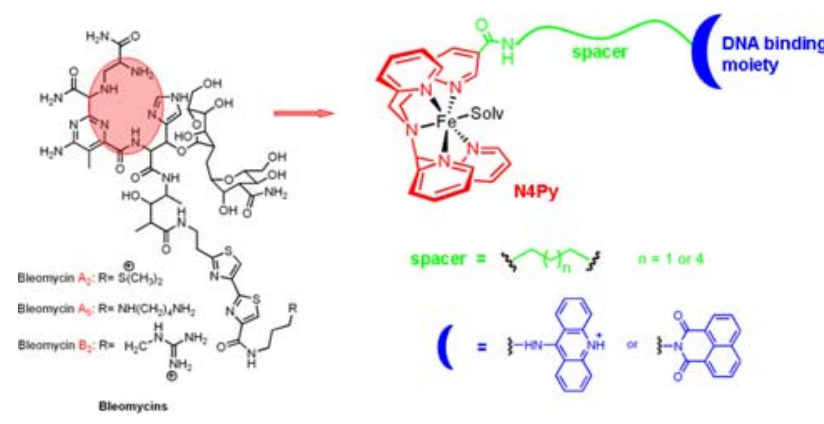

\section{References}

1. Burger RM (1998) Chem Rev 98:1153

2. Lubben M, Meetsma A, Wilkinson EC, Feringa BL, Que L Jr (1995) Angew Chem Int Ed Engl 34:1512

3. Roelfes G, Branum ME, Wang L, Que L Jr, Feringa BL (2000) J Am Chem Soc 122:11517

4. van den Berg TA, Feringa BL, Roelfes G (2007) Chem Commun 180

\section{P202}

Synthesis and properties of zinc myoglobin appending a benzenesulfonamide moiety as carbonic-anhydrase inhibitor

Asami Nakagawa, Hiroshi Takashima, Keiichi Tsukahara Department of Chemistry, Faculty of Science, Nara Women's University, Nara 630-8506, Japan. faa_nakagawa@cc.nara-wu.ac.jp Photoinduced electron-transfer reaction of hemoprotein complexes has been one of the important research subjects. We have prepared chemically modified myoglobin $(\mathrm{Mb})$, whose cofactor is replaced by zinc(II) porphyrin derivative, to construct an artificial photoinduced reaction system.

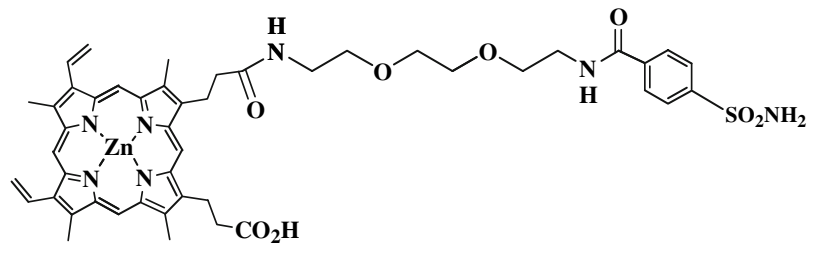

\section{ZnPPSA}

Carbonic anhydrase (CA) is a metalloenzyme which catalyses the convention of carbon dioxide to carbonate and hydrogen ions. Benzenesulfonamide derivatives have been developed as enzyme inhibitors for CA. In this study, we have synthesized zinc porphyrin having a benzenesulfonamide moiety (ZnPPSA) and reconstituted it into apoMb. ZnPPSA was synthesized via seven steps and ZnMbSA was successfully obtained by cofactor reconstitution of ZnPPSA to apoMb. The absorption spectral change of $\mathrm{ZnMbSA}$ in addition of $\mathrm{CA}$ suggests the formation of ZnMbSA-CA complex. Interactions between $\mathrm{ZnMbSA}$ and $\mathrm{CA}$ are discussed.

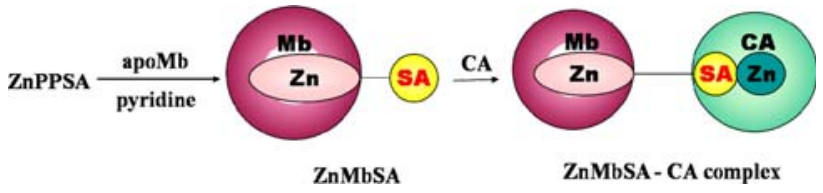

P203

Azurin-poly( $N$-isopropylacrylamide) conjugates by site-directed mutagenesis and their thermoresponsive behavior in electron transfer processes Suguru Ozawa ${ }^{1}$, Hiroshi Nakajima ${ }^{1,2,3}$, Nobuyuki Takatani ${ }^{1,2,3}$, Yoshihito Watanabe ${ }^{2}$, Nadine Rosenberger ${ }^{3}$, Armido Studer $^{3}$ ${ }^{1}$ Department of Chemistry, Graduate School of Science, Nagoya University, Nagoya 464-8602, Japan.

${ }^{2}$ Research Center for Materials Science, Nagoya University, Nagoya 464-8602, Japan.

${ }^{3}$ Organisch-Chemisches Institut, Westfälische Wilhelms-Universität, 48149 Münster, Germany. yoshi@nucc.nagoya-u.ac.jp

Protein-polymer conjugates have recently received increased attention [1]. Activity and structure of such biohybrid materials can be modulated as a function of the polymer attached. In the present study, we prepared a thermo-responsive conjugate consisting of a blue copper protein, azurin (Fig. 1a) and thermo-responsive polymer, poly( $N$-isopropylacrylamide) (PNIPAM) according to the procedure shown in Fig. 1b. Electron transfer between the conjugate (AzuPNIPAM) and redox partner, cytochrome $c(\mathrm{Cyt} c)$ showed significant change in the reaction rate at the morphology transition point $\left(32{ }^{\circ} \mathrm{C}\right)$ of PNIPAM, which is not observed for azurin wild type. Analysis on the reaction rates with Azu-PNIPAMs having different chain length revealed that the longer PNIPAM provided the more apparent change in the reaction rate presumably due to the more drastic change in the morphology. 


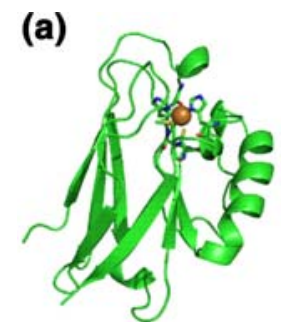

NIPAM

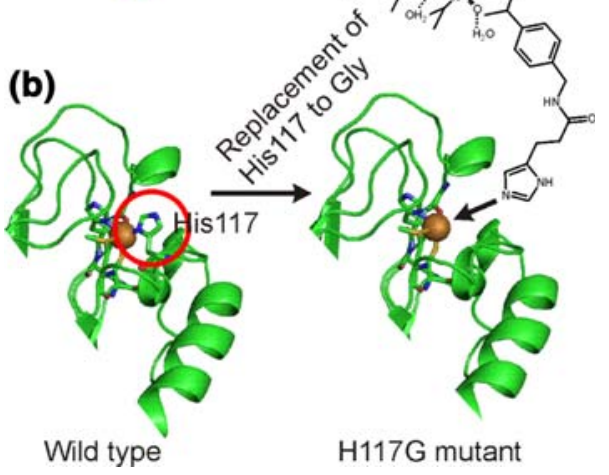

Fig. 1 a Whole structure of azurin; b introduction of PNIPAM to azurin. Replacement of His117, one of the Cu ligands in azurin, opens the coordination site for PNIPAM attached the imidazole group to the terminal

\section{Reference}

1. Rosenberger N, Studer A, Takatani N, Nakajima H, Watanabe Y (2009) Angew Chem Int Ed 48:1946-1949

\section{P204}

DNA binding by photo-controllable zinc finger peptide Akiko Nomura, Akimitsu Okamoto

Advanced Science Institute, RIKEN (The Institute of Physical and

Chemical Research), Wako, Saitama 351-0198, Japan.

a-nomura@ riken.jp

The Cys2His2-type zinc finger motifs repeatedly appear in eukaryotic transcription factors, and they help proteins bind to the DNA duplex in a sequence-specific fashion. Each finger comprises approximately 30 amino acids and independently folds into the $\beta \beta \alpha$ conformation, stabilized by a zinc ion. Zinc finger motifs have been utilized to create artificial functional proteins/peptides owing to the relatively short amino acid sequence, the well-known structure, and functions. Herein, we prepared a new artificial zinc finger peptide that has an azobenzene unit at the $\mathrm{N}$-terminus to change its structure and function upon photo-irradiation.

The azobenzene-appended zinc finger peptide was synthesized through a conventional Fmoc method. Modification at the N-terminus is structurally simple and synthetically easy, and will not affect the structure and function of the zinc finger motifs. The zinc finger domains recognize a particular target DNA sequence through two parts of interactions: contacts between nucleic bases and the $\alpha$-helix region and polar interactions between the phosphate DNA backbone and the amino acid residues in the linker and $\beta$-sheet regions. Since the former plays a crucial role in sequence-specific recognition, modification of the $\alpha$-helix region may cause undesired non-specific DNA binding. To avoid this, we have introduced an azobenzene unit at the N-terminus of the linker region of tandem zinc finger motifs. Spectroscopic studies indicated that the artificial peptide was capable of folding upon zinc binding and that photo-isomerization of the azobenzene unit did not hamper the $\alpha$-helix folding. Herein, we will also report the photo-controlled binding of the artificial zinc finger peptide to the target DNA in detail.

\section{P205}

Engineering of cytochrome $c_{552}$ derived from Thermus thermophilus toward thermally tolerant artificial peroxidase

Naomi Kawaba ${ }^{1}$, Hiroshi Nakajima ${ }^{1}$, Nobuyuki Takatani ${ }^{2}$, Yoshihito Watanabe ${ }^{2}$

${ }^{1}$ Department of Chemistry, Graduate School of Science, Nagoya University, Nagoya 464-8602, Japan.

${ }^{2}$ RCMS, Nagoya University, Nagoya 464-8602, Japan. yoshi@nucc.cc.nagoya-u.ac.jp

Application of peroxidases as catalysts for the oxidation of a wide spectrum of organic compounds has been a major focus in industrial sectors. The full-scale use of peroxidases is, however, restricted by poor thermal and organic solvent stabilities, as is the case with many commercially available enzymes. Here, we demonstrate that cytochrome $c_{552}\left(\mathrm{Cyt} c_{552}\right)$ from Thermus thermophilus HB8 can be transformed into a thermally artificial peroxidase by rational modification based on the molecular mechanism of natural peroxidase [1]. Cyt $c_{552}$ is a small electron transfer protein having $\mathrm{His}^{15}$ and $\mathrm{Met}^{69}$ as heme axial ligands. We have prepared a variant, V49D/M69A in which the original Val ${ }^{49}$ and $\mathrm{Met}^{69}$ were replaced to Asp and Ala, respectively, to introduce the active center of peroxidase to the heme cavity. V49D/M69A retains thermostability comparable to the wild type and exerts the better enzymatic activity than myoglobin H64D variant which is an engineered protein known to show a highest enzymatic activity among artificial peroxidases. Kinetic analysis for V49D/M69A revealed that the $\mathrm{Asp}^{49}$ serves as an acid-base catalyst, conforming rationality of the molecular design furnished to Cyt $c_{552}$. EPR analysis for a series of Tyr and Trp variants showed that oxoferryl-porphyrin $+\mathrm{Tyr}^{45}$ radical is a dominant active species in the peroxidase reaction by V49D/M69A. Based on this finding, additional molecular engineering aiming the higher enzymatic activity is in progress.

\section{Reference}

1. Nakajima H, Ichikawa Y, Satake Y, Takatani N, Mannna SK, Rajbongshi J, Mazumidar S, Watanabe Y (2008) ChemBioChem 9:2945-2957

\section{P206}

\section{Enhancement of thermostability of Hydrogenobacter} thermophilus cytochrome $c_{552}$ through stabilization of heme active site

Kiyofumi Irie, Hulin Tai, Shigenori Nagatomo,

Yasuhiko Yamamoto

Department of Chemistry, University of Tsukuba, Tsukuba 305-8571, Japan.irie.fcb@dmb.chem.tsukuba.ac.jp

Thermophile Hydrogenobacter thermophilus cytochrome $c_{552}$ (HT) and mesophile Pseudomonas aeruginosa cytochrome $c_{551}$ (PA) are small monoheme-containing electron transfer proteins, which are composed of 80 and 82 amino acid residues, respectively. The two proteins exhibit high sequence identity (56\%), and their main-chain folding is almost identical to each other. But HT is more stable to heat than PA. A detailed comparison of the protein interior between them highlighted sizable differences in packing among amino acid side 
chains in the protein interior, and site-directed mutants of PA, for which amino acid substitutions were selected with reference to the corresponding residues in HT, exhibited thermostabilities between those of the two proteins. We extended our efforts to stabilize protein of HT by amino acid substitutions in order to create proteins even more thermostable than HT.

Val30 is a conserved residue in both HT and PA, and is located near heme. We found that the substitution of Val30 by Ile results in increasing thermostability of protein in a fashion that denaturation temperature of oxidized HT, i.e. $108.8{ }^{\circ} \mathrm{C}$, is elevated by $\sim 4{ }^{\circ} \mathrm{C}$ in the V30I mutant. Val30 is located in hydrophobic core of the protein and V30I mutation is likely to improve packing among amino acid side chains in the core near heme. This finding demonstrated that amino acid substitutions which stabilize heme active site of cytochrome $c$ increase its thermostabilities (Fig. 1).

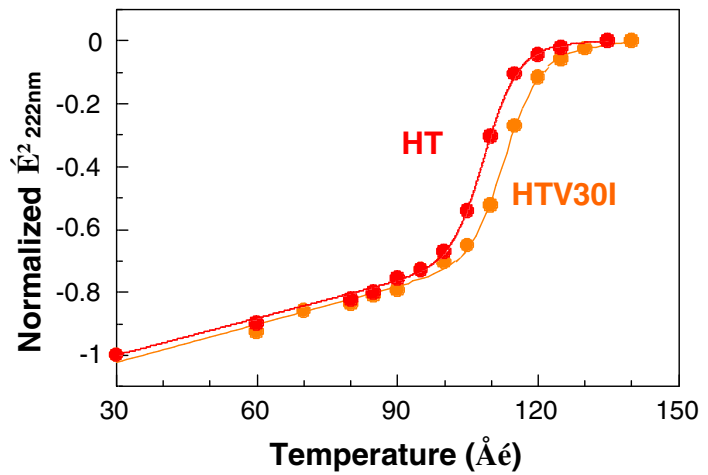

Fig. 1 Denaturation curves of oxidized forms of HT (red circles) and HTV30I (orange circles)

\section{P207}

Water-soluble nitrosyl penicillamine iron complex: a new NO donor with antitumor and hypertensive

\section{properties}

Tatiana Roudneva, Nataliya Sanina, Sergey Aldoshin

Institute of Problems of Chemical Physics, Russian Academy of

Science, Chernogolovka, Moscow Region, Russian Federation. ruta@icp.ac.ru

The discovery of the key role NO in the vasodilatation processes and growth of tumor cells stimulates researches on search of new donors of nitric oxide. At the moment, nitrosyl complexes of non-heme iron which are models of co-factors of nitrosyl $[2 \mathrm{Fe}-2 \mathrm{~S}]$ proteins are considered the most perspective in this sense.

The purpose of this work was to synthesize water-soluble binuclear tetranitrosyl iron complex with penicillamine-ligand of natural origin containing sulfur (Fig. 1). Its ability to release $\mathrm{NO}$ and antiproliferation activity in vitro in water solutions were studied and hypotensive properties were estimated. The kinetics of NO generation of complex in solid state (using mass spectroscopy method) and in water solutions (by means of a "amiNO-700" sensor produced by "inNO-T" (USA) and by mass spectrometric analysis of solutions) were studied.

Acknowledgments. This work has been performed with the financial support of the Program of the RAS "Fundamental problems of medicine" and grant of "Russian Science Support Foundation".

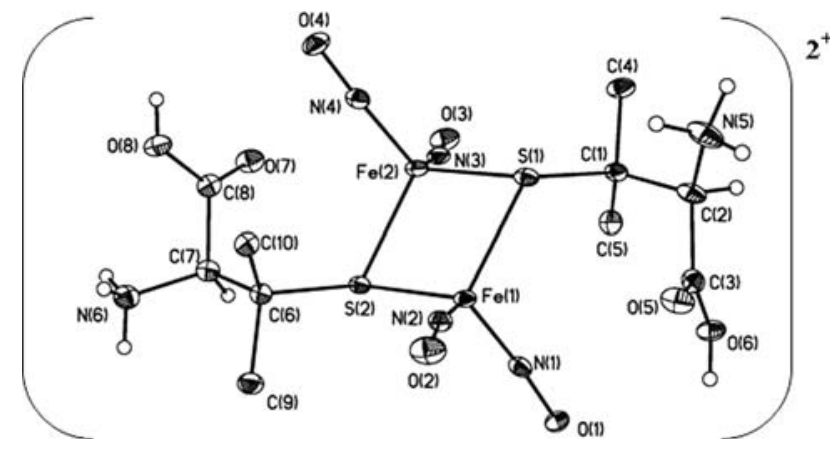

Fig. 1 The molecular structures of nitrosyl cation of complex

\section{P208}

\section{DNA-based hybrid catalysts}

\section{Gerard Roelfes}

Stratingh Institute for Chemistry, University of Groningen,

Nijenborgh 4, 9747 AG Groningen, The Netherlands.

J.G.Roelfes@rug.nl

Hybrid catalysis is an emerging approach that involves a combination of the catalytic power of transition metal complexes with the welldefined chiral structures of biopolymers. We have introduced the concept of DNA-based asymmetric catalysis [1]. The power of this concept has been demonstrated in a variety of important enantioselective copper(II) catalyzed $\mathrm{C}-\mathrm{C}$ bond forming reactions in water, including the Diels-Alder [1], Michael addition [2] and Friedel-Crafts alkylation reaction [4]; in several examples up to $99 \%$ ee was obtained.

Here also the results of a study that has revealed the special role DNA plays in these reactions will be presented: not only does DNA confer chirality to the reaction in a sequence dependent fashion, it also causes rate accelerations of up to two orders of magnitude [5].

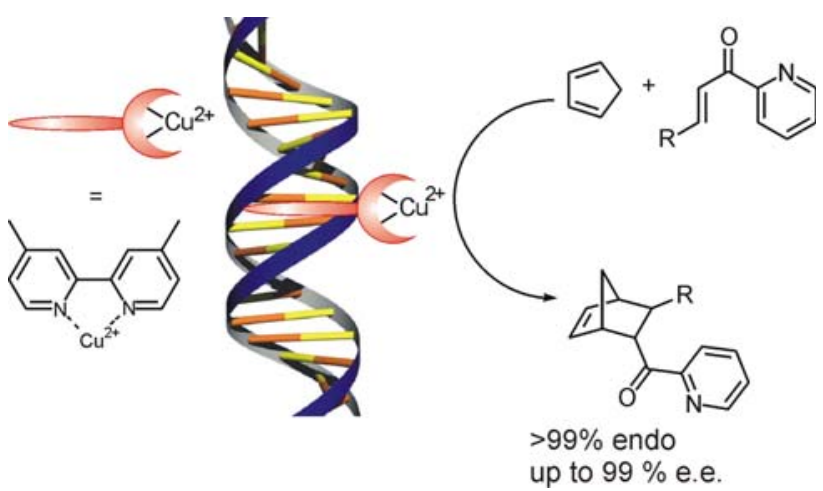

References

1. Roelfes G, Boersma AJ, Feringa BL (2006) Chem Commun 635

2. Coquière D, Feringa BL, Roelfes G (2007) Angew Chem Int Ed 46:9308

3. Boersma AJ, Feringa BL, Roelfes G (2009) Angew Chem Int Ed (in press)

5. Boersma AJ, Klijn JE, Feringa BL, Roelfes G (2008) J Am Chem Soc 130:11783 


\section{P209}

\section{Design of a catalytic $\mathrm{Ca}^{2+}$ sensor}

Ivan V. Korendovych, Daniel W. Kulp, William F. DeGrado

Department of Biochemistry and Biophysics, University of

Pennsylvania, Philadelphia, PA 19104, USA.

ivank@mail.med.upenn.edu

Linking catalytic activity to metal ion binding creates a functional sensor, which provides a number of advantages: low background noise, relative ease of function modification, amplification of the readout, etc.

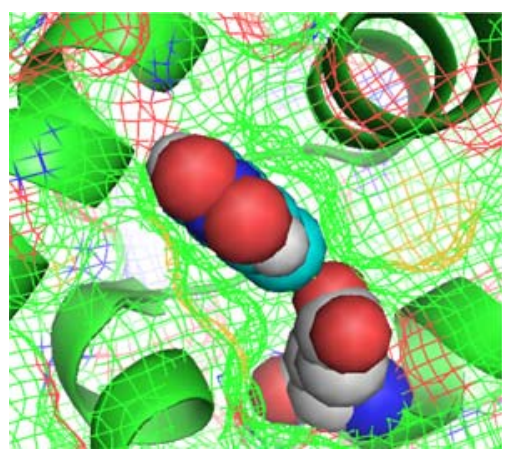

We present a catalyst that is capable of $\mathrm{C}-\mathrm{H}$ proton abstraction at neutral $\mathrm{pH}$ in a selective and calcium-dependent fashion. The activity has been achieved by placing a glutamic acid residue in a hydrophobic environment thus increasing the effective $\mathrm{p} K_{\mathrm{a}}$ of the carboxylate. We have chosen calmodulin $(\mathrm{CaM})$, a eukaryotic calcium sensor and signal transducer, as the scaffold. The CaM scaffold provides the binding pocket for the substrate and, importantly, a switchability of the function, i.e. the desired activity occurs only in the presence of $\mathrm{Ca}^{2+}$. The designed enzyme, based on the single mutation in the $\mathrm{CaM}$ hydrophobic core, accelerates Kemp elimination by at least four orders of magnitude. Further modification around the de novo created active site which enhances the activity even more.

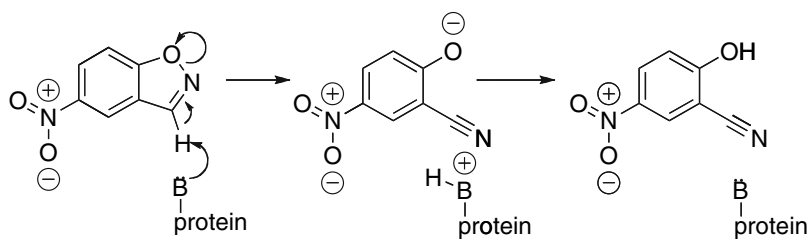

\section{P210}

\section{Controlling enantioselectivity of enzymatic sulfoxidation catalyzed by cytochrome $\mathrm{P450}_{\mathrm{BS} \beta}$ with decoy molecules}

Takashi Fujishiro ${ }^{1}$, Osami Shoji ${ }^{1}$, Shingo Nagano ${ }^{2}$, Yoshitsugu Shiro' ${ }^{2}$, Yoshihito Watanabe ${ }^{2}$

${ }^{1}$ Department of Chemistry, Graduate School of Science and Research Center for Materials Science, Nagoya University,

Nagoya 464-8602, Japan.

${ }^{2}$ RIKEN SPring-8 Center, Harima Institute, Hyogo 679-5148, Japan. yoshi@nucc.cc.nagoya-u.ac.jp

Biocatalysts have been of much interest in organic synthesis because of their high catalytic activities and stereoselectivities. The use of biocatalysts is sometimes limited due to their high substrate specificity. Although rational protein design by site-directed mutagenesis has been demonstrated to be an effective method to change the substrate specificity of enzymes, it is impossible to always obtain desired mutants. In addition, preparation and purification of mutants are still a time-consuming process. Here, we report a simple method to change the substrate specificity of cytochrome $\mathrm{P} 450_{\mathrm{BS} \beta}\left(\mathrm{P}_{450} \mathrm{BS}_{\beta}\right)$ using decoy molecules (carboxylic acids), a fake molecule of its substrate [1]. Interestingly, the enantioselectivity of sulfoxidation of thioanisole was also altered by changing the decoy molecules. With heptanoic acid as a decoy molecule, the $R$-isomer of the sulfoxide was obtained as the main product ( $29 \%$ ee). On the other hand, when $p$-methylphenylacetic acid was used as decoy molecule, the $S$-isomer was obtained (24\% ee).

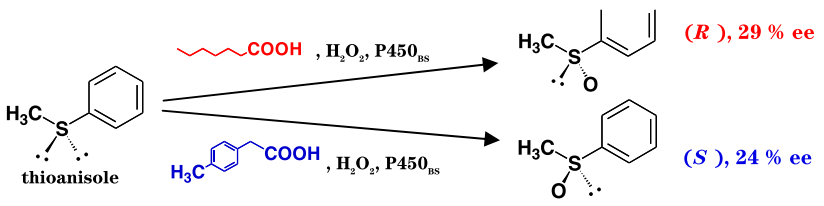

\section{Reference}

1. Shoji O, Fujishiro T, Nakajima H, Kim M, Nagano S, Shiro Y, Watanabe Y (2007) Angew Chem Int Ed 46:3656-3659

\section{P211}

Electronic structure, properties and modulation of iron displacements in a saddle distorted porphyrin macrocycle and implications for the hemoproteins

\section{Sankar Prasad Rath* and Ranjan Patra}

Department of Chemistry, Indian Institute of Technology Kanpur, Kanpur 208016, India.

sprath@iitk.ac.in

A rare family of five and six-coordinated high-spin Fe(III) porphyrins incorporating axial ligands is synthesized and structurally characterized which demonstrates, for the first time, step-wise metal displacements in a single distorted macrocyclic environment that has generally been seen in many biological systems. The introduction of four nitro groups into the meso-positions of octaethyl porphyrin provides an interesting modulation of the macrocycle properties, which enables the facile isolation of 'pure' high-spin FeIII $(t n$ $\mathrm{OEP}) \mathrm{Cl}, \mathrm{FeIII}(t n-\mathrm{OEP})(\mathrm{MeOH}) \mathrm{Cl}$ and $\mathrm{FeIII}(t n-\mathrm{OEP})\left(\mathrm{OH}_{2}\right)_{2} \mathrm{ClO}_{4}$ in excellent yields in a saddle distorted macrocyclic environment. Moreover, the addition of axial ligand $\mathrm{L}$ to $\mathrm{FeIII}(t n-\mathrm{OEP}) \mathrm{Cl}$ in air immediately auto reduce to air-stable $\mathrm{FeII}(t n-\mathrm{OEP})(\mathrm{L})_{2}$ which also exhibits the hallmarks of a ferric porphyrins in the solid. The porphyrin core is least distorted in $\mathrm{FeIII}(t n-\mathrm{OEP})\left(\mathrm{H}_{2} \mathrm{O}\right)_{2} \mathrm{ClO}_{4}$ with highest core size $(2.061 \AA)$ while the core is highly distorted with lowest core size $(1.962 \AA)$ in $\mathrm{FeII}(t n-\mathrm{OEP})(3-\mathrm{Cl}-\mathrm{py})_{2}$ thus authenticates a significant and unprecedented core expansion of $0.1 \AA$ in the series.

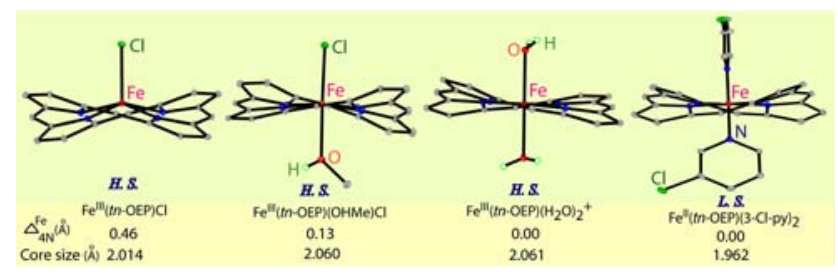

\section{Reference}

1. Patra R, Chaudhury A, Ghosh SK, Rath SP (2008) Inorg Chem $47: 8324-8335$ 


\section{P212}

Azurin-poly( $N$-Isopropylacrylamide) conjugates by site directed mutagenesis and their thermo-responsive behavior in electron transfer processes

Suguru Ozawa ${ }^{1}$, Hiroshi Nakajima, Nobuyuki Takatani, Yoshihito Watanabe $^{2}$, Nadine Rosenberger ${ }^{3}$, Armido Studer $^{3}$

${ }^{1}$ Department of Chemistry, Graduate School of Science Nagoya University, Nagoya 464-8602, Japan.

${ }^{2}$ R CM Science, Nagoya University, Nagoya 464-8602, Japan

${ }^{3}$ Organisch-Chemisches Institute, Westfälische Wilhelms-University, 48149 Münster, Germany.

yoshi@nucc.nagoya-u.ac.jp

Protein-polymer conjugates have recently received increased attention [1]. Activity and structure of such biohybrid materials can be modulated as a function of the polymer attached. In the present study, we prepared a thermo-responsive conjugate consisting of a blue copper protein, azurin (Fig. 1a) and thermo-responsive polymer, poly( $N$-Isopropylacrylamide)(PNIPAM) according to the procedure shown in Fig. 1b. Electron transfer between the conjugate (AzuPNIPAM) and redox partner, cytochrome $c$ (Cyt $c$ ) showed significant change in the reaction rate at the morphology transition point $\left(32{ }^{\circ} \mathrm{C}\right)$ of PNIPAM, which is not observed for azurin wild type. Analysis on the reaction rates with Azu-PNIPAMs having different chain-length revealed that the longer PNIPAM provided the more apparent change in the reaction rate presumably due to the more drastic change in the morphology.
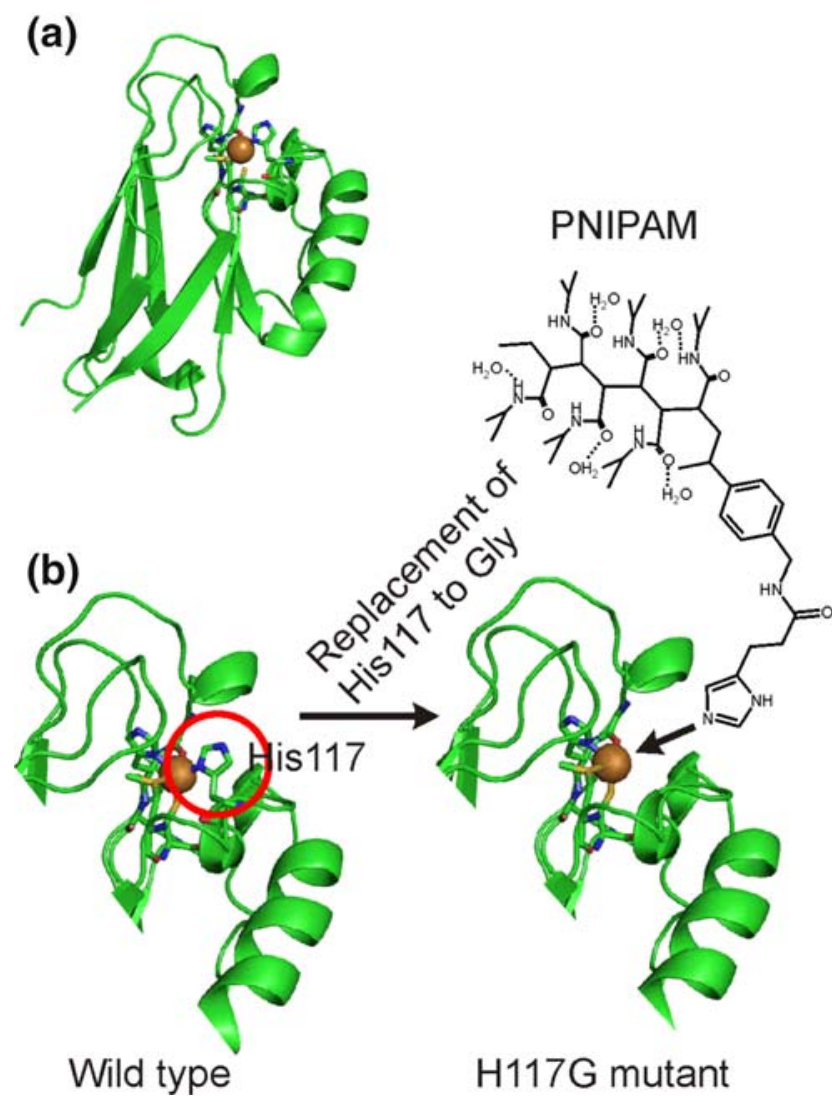

Fig. 1 a Whole structure of azurin; b introduction of PNIPAM to azurin. Replacement of His117, one of the $\mathrm{Cu}$ ligands in azurin, opens the coordination site for PNIPAM attached the imidazole group to the terminal

\section{References}

1. Rosenberger N, Studer A, Takatani N, Nakajima H, Watanabe Y (2009) Angew Chem Int Ed 48:1946-1949

\section{P213}

Rational design of catalytically relevant metalloproteins: synthesis and characterization of molybdenum-sulfur analogues of iron-sulfur proteins

J. Kristensen ${ }^{1}$, B. L. Ooi ${ }^{1}$, W. R. Hagen ${ }^{2}$, H.E.M. Christensen ${ }^{1}$

${ }^{1}$ Department of Chemistry, Technical University of Denmark, 2800 Kgs. Lyngby, Denmark.

${ }^{2}$ Department of Biotechnology, Delft University of Technology, 2628 BC Delft, Netherlands.

jytte@kemi.dtu.dk

Iron-sulfur proteins with cuboidal $\left[\mathrm{Fe}_{3} \mathrm{~S}_{4}\right]$ and $\left[\mathrm{Fe}_{4} \mathrm{~S}_{4}\right]$ clusters are interesting due to the variation of reduction potentials and accessible oxidation states they display [1]. Synthetic inorganic $\left[\mathrm{Mo}_{4} \mathrm{~S}_{4}\right]$ clusters are similar in size to the $\mathrm{Fe}$ analogue and possess interesting properties [2] that make them attractive candidates for active site re-design of iron-sulfur proteins. We have synthesized and purified the molybdenum-sulfur analogue of the ferredoxin from Pyrococcus furiosus. Mass spectrometric studies of the purified products have confirmed the successful incorporation of the molybdenum-sulfur cluster into the protein. Two closely related species have be purified and investigated by UV-visible spectroscopy, mass spectrometry and EPR spectroscopy. The EPR studies suggest stabilization of multiple oxidation states of the molybdenum-sulfur cluster by the protein.

\section{References}

1. Johnson MK (1998) Curr Opin Chem Biol 2:173-181

2. Shibahara T, Kuroya H, Akashi H, Matsumoto K, Ooi S (1993) Inorg Chim Acta 212:251-263

\section{P214}

The site of the tricopper cluster in the particulate methane monooxygenase (pMMO)

Marianne M. Lee ${ }^{1}$, I-Jui Hsu ${ }^{2,3}$, Feng-Chun Lo $^{3}$, Jeff C.-H. Lai ${ }^{3}$, Kok Yaoh $\mathrm{Ng}^{3}$, Terra Y.-J. Lu ${ }^{3}$, Steve S.-F. Yu ${ }^{3}$, Michael K. Chan ${ }^{1}$, and Sunney I. Chan ${ }^{2,3}$

${ }^{1}$ Department of Chemistry and Biochemistry, Ohio State University, Columbus, OH 43210, USA.

${ }^{2}$ Department of Chemistry, National Taiwan University, Taipei 10617, Taiwan.

${ }^{3}$ Institute of Chemistry, Academia Sinica, Nankang, Taipei 11529, Taiwan.

sunneychan@yahoo.com

A fusion protein containing the peptide sequence HIQAMLTMGDWD binds three $\mathrm{Cu}$ (II) ions cooperatively to form a tricopper cluster. This peptide was constructed from the hydrophilic cluster of the potential metal-ligating residues that lined the "empty" cavity of Site D in the transmembrane domain of the crystal structure of the particulate methane monooxygenase (pMMO) reported by Rosenzweig and coworkers [1]. $\mathrm{Cu} \mathrm{K}$-edge EXAFS measurements revealed a ligand structure for the tricopper cluster that is consistent with the structure of the $\mathrm{Cu}^{\mathrm{II}} \mathrm{Cu}^{\mathrm{II}} \mathrm{Cu}^{\mathrm{II}}$ triad with capping "oxo" modeled into Site D by the Chan laboratory [2]. $77 \mathrm{~K}$ EPR indicated that the three $\mathrm{Cu}(\mathrm{II})$ ions in this complex are ferromagnetically coupled with $S_{\mathrm{T}}=3 / 2$, as observed in pMMO-enriched membranes and pMMO isolated from Methylococcus capsulatus (Bath) with the full complement of $\sim 15$ copper ions [3]. Studies of the reduction of the tricopper cluster with benzoin and reoxidation of the reduced cluster will be described.

\section{References}

1. Lieberman RL, Rosenzweig AC (2005) Nature 434:177-182 
2. Chan SI, Wang VC-C, Lai JC-H, Yu SS-F, Chen PP-Y, Chen KHC, Chen C-L, Chan MK (2007) Angew Chem Int Ed 46:1992-1994

3. Chan SI, Yu SS-F (2008) Acc Chem Res 41:969-979

\section{P215}

Models of noncoupled dinuclear copper centers in azurin

Steven M. Berry, Oleksandra Kniazieva, Jonathan R. Mayers, Nicholas A. Zehm

Department of Chemistry and Biochemistry, University of Minnesota Duluth, Duluth, MN 55812, USA.

smberry@d.umn.edu

Modeling of metalloproteins is an important facet of understanding their structure and function. Furthermore, metalloprotein design, or the design of defined metal binding sites into a protein, is an important and growing field with much future promise. Toward these goals, models of the noncoupled copper centers found in the enzymes peptidyl $\alpha$-hydroxylating monooxygenase (PHM), dopamine $\beta$-monooxygenase (DBM), and nitrite reductase (NiR) were designed into the small soluble protein azurin. The models are significant because they maintain the existing type 1 (T1) copper, electron transfer site of azurin while including the second designed type 2 (T2) copper center that mimics the $\mathrm{T} 2$ catalytic sites in the target enzymes. UV-vis absorption and EPR spectroscopy data of the model sites are consistent with $\mathrm{T} 2$ centers and establish copper binding at the sites, thus modeling those found in PHM/DBM and NiR. Importantly, the models' approximate 11-13 $\AA$ separation between the T1 and T2 copper sites is comparable with the separations in the native systems. Recent evaluation of activity and further studies on these and related azurin models will be presented.
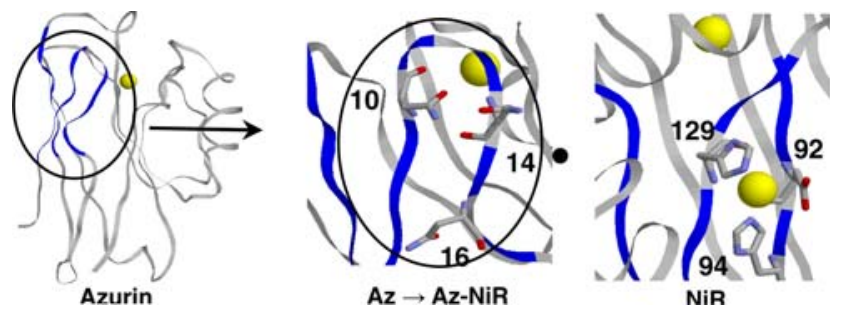

Reference

1. Berry SM, Mayers JR, Zehm NA (2009) J Biol Inorg Chem 14(1):143-149

\section{P216}

Artificial diiron center in dioxygen-binding protein Akira Onoda $^{1,2}$, Yasunori Okamoto ${ }^{1}$, Takashi Hayashi ${ }^{1}$ ${ }^{1}$ Department of Chemistry, Graduate School of Engineering Osaka University, Suita 565-0871, Japan.

${ }^{2}$ Frontier Research Base for Global Young Researchers, Graduate School of Engineering Osaka University, Suita 565-0871, Japan. onoda@chem.eng.osaka-u.ac.jp

Diiron center is found in vast range of metalloproteins, which perform a variety of functions. Members of this superfamily has potential for oxidation of hydrocarbon in methane monooxygenase, peroxide scavenging in rubrerythrin, radical generation in ribonucleotide reductase, iron storage in ferritin and $\mathrm{O}_{2}$ transport in hemerythrin. All these proteins contain dinuclear iron core supported by four-helix bundle. The proteins activating $\mathrm{O}_{2}$ molecules have diiron site surrounded by carboxylate-rich environment, such as (His)2(Asp/Glu) 4 or His2(Asp/Glu)4, whose imidazole groups located at the opposite side of the dioxygen molecule coordinating to iron. One of typical protein in this group, methane monooxygenase hydroxylase, generates the peroxo intermediate, followed by the key intermediate Q. While dioxygen-binding proteins, hemerythrin family, contain the diiron core coordinated with His5(Asp/Glu)2 residues, which perform reversible dioxygen binding. Modulating the coordination sphere of diiron proteins is of great interest from the view of elucidation on the mechanistic insight, as well as harnessing non-native properties and activities.

We focused on hemerythrin domain located in the bacterial chemotaxis protein of Desulfovibrio vulgaris (DcrH), which consist of non-heme diiron center analogous to the family of the hemerythrin protein found in marine invertebrates. This domain, DcrH-Hr, is thought to be involved in dioxygen binding and sensing [1]. To modulate their inherent dioxygen binding function, we prepared several mutants of DcrH-Hr with different coordination environment; the properties of the mutants will be discussed.

\section{Reference}

1. Xiong J, Kurtz DM Jr, Ai J, Sanders-Loehr J (2000) Biochemistry 39:5117-5125

\section{P217}

Novel protein catalysts containing multinuclear metal cluster Yohei Sano $^{1}$, Rie Sakurai ${ }^{1}$, Akira Onoda ${ }^{1,2}$, Takashi Hayashi ${ }^{1}$ ${ }^{1}$ Department of Chemistry, Graduate School of Engineering Osaka University, Suita 565-0871, Japan.

${ }^{2}$ Frontier Research Base for Global Young Researchers, Graduate School of Engineering Osaka University, Suita 565-0871, Japan.

ysano@chem.eng.osaka-u.ac.jp

Metalloproteins containing multinuclear cluster catalyze a wide variety of reduction, oxidation, and multiple electron transfer in nature. Fascinating paramount of this class of proteins is nitrogenase (109 kDa) having $\mathrm{MoFe}_{7} \mathrm{~S}_{9} \mathrm{X}$ cluster (FeMo-cofactor), which converts $\mathrm{N}_{2}$ to $\mathrm{NH}_{3}$ in aqueous solution under ambient conditions. However, nitrogenase is huge and unstable to atmospheric oxidation. As a new approach of creating artificial metal catalysts, we focused on "metallothionein" (MT), which is small (6 kDa) cysteine-rich protein involved in metal storage and detoxification and is potential for formation of stable metal clusters.

As a protein matrix, we designed the $\alpha$-domain of MT (MT $\alpha$ ) and its mutant, MT $\alpha$-Cys29Ala, which would provide an effective vacant site for substrate binding. These peptides were synthesized by solidphase methods with Fmoc chemistry. Successful formation of tetranuclear $\mathrm{Co}^{\mathrm{II}}$ and $\mathrm{Fe}^{\mathrm{II}}$ cores using these proteins was confirmed by $\mathrm{UV}-\mathrm{vis}$ in aqueous solution under $\mathrm{N}_{2}$ atmosphere. Titration experiments of MT $\alpha$ with $\mathrm{Co}^{\mathrm{II}}$ ions showed that the absorption intensity of LMCT transitions ( 329 and $415 \mathrm{~nm}$ ) and characteristic $d-d$ transitions $(600,690$, and $743 \mathrm{~nm})$ increased linearly with the $\mathrm{Co}^{\mathrm{II}} / \mathrm{MT} \alpha$ ratio up to 4 equiv of $\mathrm{Co}^{\mathrm{II}}$ ions, indicating successful tetrahedral $\mathrm{Co}^{\mathrm{II}} \mathrm{S}_{4}$ coordination in $\mathrm{Co}_{4}{ }_{4}$ core. Furthermore, the molecular mass of the $\mathrm{Co}^{\mathrm{II}}{ }_{4}$-MT $\alpha$ was also determined by ESI-TOF MS. We have prepared tetranuclear $\mathrm{Co}^{\mathrm{II}}$ and $\mathrm{Fe}^{\mathrm{II}}$ cores of these two protein matrices, MT $\alpha$ and MT $\alpha$-Cys29Ala, and the catalytic activities of these metal clusters were investigated using reduction of hydrazine and analogous derivatives as a model reaction for dinitrogen reduction.

\section{P218}

De novo design and characterization of a novel 3-His di-iron carboxylate enzyme

Amanda J. Reig, Daniel W. Kulp, William F. DeGrado

Department of Biochemistry and Biophysics, University of Pennsylvania, Philadelphia, PA 19104, USA.

reig@mail.med.upenn.edu

The recent de novo design of a single-chain di-iron carboxylate fourhelix bundle protein (DFsc) has provided the groundwork for investigations into how Nature tunes the metal-binding specificity and catalytic activity of natural di-iron oxidation enzymes [1]. As an 
extension of this work, we have successfully modified the DFsc scaffold to incorporate a third active site His residue, similar to that found in the natural oxygenase, AurF. Our 3-His DFsc enzyme successfully binds Fe, activates dioxygen, and possesses the ability to catalyze the oxidation of $p$-anisidine to an azobenzene adduct. This work represents one of the first examples of a de novo designed metalloenzyme in which fine tuning of the active site cavity successfully altered the catalytic functionality.
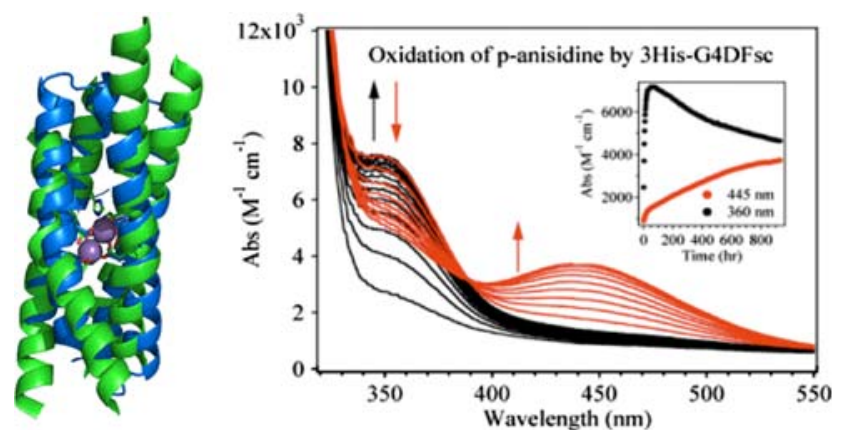

Reference

1. Calhoun JR, Kono H, Lahr S, Wang W, DeGrado WF, Saven JG (2003) J Mol Biol 334:1101-1115 